International Journal of Engineering \& Technology, $7(4.30)(2018)$ 160-164
International Journal of Engineering \& Technology
WPC
Website: www.sciencepubco.com/index.php/IJET
Research paper

\title{
Fuzzy Finite Switchboard Automata with Complete Residuated Lattices
}

\author{
Nur Ain Ebas ${ }^{1}$, Nor Shamsidah Amir Hamzah ${ }^{1 *}$, Kavikumar Jacob ${ }^{1}$, Mohd Saifullah Rusiman ${ }^{1}$ \\ ${ }^{1}$ Department of Mathematics and Statistics, Faculty of Applied Science and Technology, Universiti Tun Hussein Onn \\ Malaysia, Kampus Pagoh, 84600, Pagoh, Muar, Johor, Malaysia \\ *Corresponding author E-mail:shamsida@uthm.edu.my
}

\begin{abstract}
The theory of fuzzy finite switchboard automata (FFSA) is introduced by the use of general algebraic structures such as complete residuated lattices in order to enhance the process ability of FFSA. We established the notion of homomorphism, strong homomorphism and reverse homomorphism and shows some of its properties. The subsystem of FFSA is studied and the set of switchboard subsystemforms a complete $\mathcal{L}$-sublattices is shown. The algorithm of FFSA with complete residuated lattices is given and an example is provided.
\end{abstract}

Keywords: Complete Residuated Lattices; Fuzzy Finite Automata; Fuzzy Finite Switchboard Automata; Switchboard Subsystems.

\section{Introduction}

A finite state machine is a mathematical computation model that is used to design the computer programs along with sequential logic circuits. Many researchers have studied the idea of finite automata since 1940. The theory of automata had investigated by Rabin and Scott [7]. Wee [23] is the first researcher who introduced the concept of the theory of automata in the fuzzy environment. In general fuzzy finite state machine (FFSM) or fuzzy finite automata (FFA) has membership grades in an interval $[0,1]$ but there is a possibility to extended the membership values into more general algebraic structures. Qiu studied those so-called theories and their characterizations where he considered its membership grades under the fact of complete residuated lattices $[17,20]$. In the following year, Qiu extended his research into specific type of automata which are pushdown automata, turning machine and reduction and minimization $[5,9,17]$. Many researchers studied on fuzzy finite automata with membership value in a Complete Residuated Lattices (CRL) [2,4,9,11,13,14,15,17,19,20,21,22]. As a continuation of the FFA, the concept of fuzzy finite switchboard state machine (FFSSM) that is made up of switching and commutative state machines has been studied by Sato and Kuroki $[3,6]$. This classified fuzzy finite automaton has a mechanism that will act as a controller during the transition between the current state and next.

In this research, a complete residuated lattice is chosen because it offers the general algebraic structures associated with several important logics $[1,12,17,18]$. According to literature, the CRL has not been applied to FFSA. Therefore, in this research, the theory of FFSA is extended to a more comprehensive structure by considering the membership values in a complete residuated lattice.

\section{Preliminaries}

"An algebraic structure with strong connections to mathematical logic is known as a residuated lattice.

Definition 2.1 [24] The algebra $\mathcal{L}=(\boldsymbol{L}, \wedge, \vee, \bigotimes, \rightarrow, \mathbf{0}, \mathbf{1})$ should satisfying three conditions:

a) $(L, \wedge, \vee, 0,1)$ is a lattice with the least element is 0 and the greatest element is 1

b) $(L, \otimes, \vee)$ is a commutative monoid with the unit 1 ,

c) $\otimes$ and $\rightarrow$ form an adjoint pair.

For example, they satisfy the adjunction property: for all $x, y, z \in L, x \otimes y \Leftrightarrow x \leq y \rightarrow z$.

Let $\mathcal{L}=(L, \wedge, \vee, \otimes, \rightarrow, 0,1)$ where $\mathcal{L}$ is called complete residuated lattice if $(L, \wedge, \vee, 0,1)$ is a complete lattice, $\otimes$ is called a multiplication, $\rightarrow$ is a residuum, $\wedge$ and $\vee$ is supremum and infimum respectively. Multiplication, $\otimes$ and residuum, $\rightarrow$ are intended for modeling the conjunction and implication of the corresponding logical calculus. Meanwhile, Supremum $\vee$ and infuimum $\wedge$ are intended to model the general and existential quantifier.

The notion $x \Leftrightarrow y$ (biimplication) can be written as $(x \rightarrow y) \wedge$ $(y \rightarrow x), x \rightarrow y=\min (1-x+y, 1)$ is a complete residuated lattice while $x \otimes y=\max (x+y-1,0)$ is Standard Lukasiewcz algebra. Heyting algebra is a residuated lattice that satisfies $x \otimes$ $y=x \wedge y$. Meanwhile, the notion of Standard Godel algebra is $x \otimes y=\min (x, y)$ and $x \rightarrow y=1$ if $x \leq y$ and $y$ otherwise is a Heyting algebra [13]. There are some properties of complete residuated lattice in the following lemma:

Lemma $2.2[17,13]$ Let $\mathcal{L}$ be a complete residuated lattice. Then $\boldsymbol{x}, \boldsymbol{y}, \boldsymbol{z} \in \boldsymbol{L}$ and $\left\{\boldsymbol{x}_{\boldsymbol{i}}\right\}_{\boldsymbol{i} \in \boldsymbol{I}},\left\{\boldsymbol{y}_{\boldsymbol{i}}\right\}_{\boldsymbol{i} \in \boldsymbol{I}} \subseteq \boldsymbol{L}$ the following properties hold:

$$
\text { 1) } x \leq y \text { if and only if } x \rightarrow y=1
$$


2) $y \leq z \Rightarrow x \otimes y \leq x \otimes z$

3) $(x \leftrightarrow y) \otimes(y \leftrightarrow z) \leq x \leftrightarrow z$

4) $x \otimes \mathrm{V}_{i \in I} y_{i}=\mathrm{V}_{i \in I}\left(x \otimes y_{i}\right)$

5) $x \otimes \bigwedge_{i \in I} y_{i}=\bigwedge_{i \in I}\left(x \otimes y_{i}\right)$

6) $\vee_{i \in I} x_{i} \rightarrow y=\bigwedge_{i \in I}\left(x_{i} \rightarrow y\right)$

7) $x \rightarrow \bigwedge_{i \in I} y_{i}=\bigwedge_{i \in I}\left(x \rightarrow y_{i}\right)$

8) $\vee_{i \in I}\left(x \rightarrow y_{i}\right) \leq x \rightarrow \bigvee_{i \in I} y_{i}$

9) $\vee_{i \in I}\left(x_{i} \rightarrow y\right) \leq \wedge_{i \in I} x_{i} \rightarrow y$

10) $\bigwedge_{i \in I}\left(x_{i} \leftrightarrow y_{i}\right) \leq \bigwedge_{i \in I} x_{i} \leftrightarrow \bigwedge_{i \in I} y_{i}$

11) $\bigvee_{i \in I}\left(x_{i} \leftrightarrow y_{i}\right) \leq \bigvee_{i \in I} x_{i} \leftrightarrow \bigvee_{i \in I} y_{i}$

12) $x \leftrightarrow y \leq(x \otimes z) \leftrightarrow(y \otimes z)$.

Let $\mathcal{M}=(Q, X, \mu)$ be a FFA [8], where $Q$ and $X$ are finite non-empty sets and $\mu$ is a fuzzy subset of $\mu: Q \times X \times Q \rightarrow$ $[0,1]$, where $Q$ is called the set of states, $X$ is called the set of inputs and $\mu$ is called the transition function. Let $X^{*}$ be the set if all word elements of $X$ of finite length. Let $\beta$ be the empty words in $X^{*}$ and $|x|$ be the length of finite length. Define $\mu^{*}: Q \times X^{*} \times Q \rightarrow[0,1]$ by

and $\forall b \in X^{*}, x \in X$,

$$
\mu^{*}(q, \beta, p)= \begin{cases}1, & q=p \\ 0, & q \neq p\end{cases}
$$

$$
\mu^{*}(q, x b, p)=\vee\{\mu(q, x, r) \wedge \mu(r, b, p): r \in Q\}
$$

$\mathcal{M}$ is called switching and commutative if and only if $\mu(q, a, p)=$ $\mu(p, a, q)$ and $\mu(q, a b, p)=\mu(q, b a, p)$ for all $q, p \in Q$ and $a, b \in X$. If $\mathcal{M}$ is switching and commutative, then $\mathcal{M}$ is called $a$ fuzzy finite switchboard automata."

Definition 2.3 [20] Let $\mathcal{L}$ be a complete residuated lattices and $X$ be an (finite) alphabet. A fuzzy automaton over $\mathcal{L}$ and $X$, or simply a fuzzy automaton is a quadruple $\mathcal{M}=(Q, \delta, \sigma, \tau)$, where

a) $Q$ is a non-empty set, called the finite set of states,

b) $\delta: Q \times X \times Q \rightarrow L$ is a fuzzy subset of $Q \times X \times Q$, called the fuzzy transition function,

c) $\sigma: Q \rightarrow L$ is a fuzzy subsets of $Q$, called the fuzzy set of initial states,

d) $\tau: Q \rightarrow L$ is a fuzzy subsets of $Q$, called the fuzzy set of terminal states.

We can interpret $\delta(q, x, p)$ as the degree to which an input letter $x \in X$ causes a transition from a state $q \in Q$ into a state $p \in Q$, whereas we can interpret $\sigma(q)$ and $\tau(q)$ as the degrees to which $q$ is respectively an input state and a terminal state. We will always assume that the input alphabet $X$ is finite, but from methodological reasons we will allow the set of states $Q$ to be infinite. A fuzzy automaton whose set of states is finite is called a finite fuzzy automaton.

Definition 2.4 [20] Let $X^{*}$ denote the free monoid over the alphabet $X$. The mapping $\delta$ can be extended up to a mapping $\delta^{*}: Q \times X^{*} \times Q \rightarrow L:$

If $q, p \in Q$ and $e \in X^{*}$ is the empty word, then

$$
\delta^{*}(q, e, p)=\left\{\begin{array}{cc}
1 & q=p \\
0 & \text { otherwise }
\end{array}\right.
$$

$$
\delta^{*}(q, u x, p)=\bigvee_{r \in Q}\left(\delta^{*}(q, u, r) \otimes \delta(r, x, p)\right) .
$$

We have that for all $q, p \in Q$ and $u, v \in X^{*}$,

$$
\delta^{*}(q, u v, p)=\bigvee_{r \in Q}\left(\delta^{*}(q, u, r) \otimes \delta(r, v, p)\right)
$$

In a way that if for any $u \in X^{*}$ we define a fuzzy relation $\delta_{u}$ on $Q$ by $\delta_{u}(q, p)=\delta_{*}(q, u, b), \forall q, p \in Q$ called the fuzzy transition relation determined by $u$, then equation (1) can be written as

$$
\delta_{u v}=\delta_{u} \circ \delta_{v}, u, v \in X^{*} .
$$

Definition 2.5 [10] The switching fuzzy automaton $\overline{\mathcal{M}}=$ $(\bar{Q}, \bar{\delta}, \bar{\sigma}, \bar{\tau})$ of a fuzzy automaton $\mathcal{M}=(Q, \delta, \sigma, \tau)$ whose fuzzy transition function and fuzzy sets of initial and terminal states are defined by

$$
\bar{\delta}(q, x, p)=\delta(p, x, q)
$$

for all $q, p \in Q$ and $x \in X, \bar{\sigma}=\tau$ and $\bar{\tau}=\sigma$. In other words,

$$
\bar{\delta}_{x}=\left(\delta_{x}\right)^{-1}
$$

for each $x \in X$

\section{Remark 2.6}

- Roughly speaking the switching or reversing fuzzy automaton $\overline{\mathcal{M}}$ is obtained from $\mathcal{M}$ by exchanging fuzzy sets of initial and final states and "reversing" all the transitions.

- Due to the fact that the multiplication $\otimes$ is commutative.

Let $\mathcal{S}=(S, \oplus, \otimes, 0,1)$ be a semiring with the zero 0 and the identity 1 . Let $\mathcal{F}(Q)=\left(L^{Q}, \wedge, \vee, \emptyset, Q\right)$ is a complete lattice with the least element $\emptyset$ and the greatest element $Q$. For any $\lambda \in L$ and $f \in$ $L^{Q}$, let us define the left scalar multiplication $\lambda f$ and the right scalar multiplication $f \lambda$ as follows: $\lambda f(q)=\lambda \otimes f(q)$ and $f \lambda(q)=f(q) \otimes \lambda$, for every $q \in Q$. Due to commutativity of the multiplication $\otimes$, the left and right scalar multiplications coincide, i.e., $\lambda f=f \lambda$, for all $q \in Q$. The lattice $\mathcal{F}(Q)$ equipped with this scalar multiplication will be denoted by $\mathcal{F}_{\otimes}(Q)$ and called the $\mathcal{L}$ lattice of fuzzy subsets of the set $Q$. Any subset of $L^{Q}$ which is closed under scalar multiplication and arbitrary meets and joins, and it contains the least and the greatest element of $\mathcal{F}(Q)$ will be called a complete $\mathcal{L}$-sublattice of $\mathcal{F}_{\otimes}(Q)[10]$ "

\section{Fuzzy Finite Switchboard Automata with Complete Residuated Lattices}

Refer to 5.2.1 example, each states have multi-membership value which means transition to the same state have more than one input symbols in the same current state. Thus, in order to obtain the optimal membership value, complete residuated lattice is used. The purpose of using complete residuated lattice is to choose which is the best membership value at each transition.

Definition 3.1 Let $\mathcal{M}=(\boldsymbol{Q}, \boldsymbol{\delta}, \boldsymbol{\sigma}, \boldsymbol{\tau})$ be a fuzzy automata over $\mathcal{L}$ and $\boldsymbol{X}$.

1) $\mathcal{M}$ is called switching if and only if

$$
\boldsymbol{\delta}_{\boldsymbol{x}}(\boldsymbol{q}, \boldsymbol{p})=\left(\boldsymbol{\delta}_{x}\right)(\boldsymbol{p}, \boldsymbol{q}) \text { for } \forall \boldsymbol{q}, \boldsymbol{p} \in \boldsymbol{Q} \text { and } \boldsymbol{x} \in \boldsymbol{X}
$$

2) $\boldsymbol{M}$ is called commutative if and only if

$$
\boldsymbol{\delta}_{x y}(\boldsymbol{p}, \boldsymbol{q})=\boldsymbol{\delta}_{y x}(\boldsymbol{p}, \boldsymbol{q}) \text { for } \forall \boldsymbol{q}, \boldsymbol{p} \in \boldsymbol{Q} \text { and } \boldsymbol{x}, \boldsymbol{y} \in \boldsymbol{X} .
$$

If $\mathcal{M}$ is switching and commutative, then $\mathcal{M}$ is called fuzzy finite switchboard automata over $\mathcal{L}$ and $\boldsymbol{X}$.

Now we have the following results. The proof of the next three theorems are straightforward.

Theorem 3.2 The following conditions are equivalent:

(i). $(L, \otimes, 1)$ is an $\mathcal{L}$-monoid, that is to say, the multiplication is distributive to finite joins, $x \otimes(y \vee z)=(x \otimes y) \vee(x \otimes$ $z),(y \vee z) \otimes x=(y \otimes x) \vee(z \otimes x)$.

(ii). For any $\mathcal{M}$ over $\mathcal{L}$ and $X$ and for any $q, p \in Q$ and $x, y \in$ $X^{*}, \delta_{x y}^{*}(q, p)=\delta_{y x}^{*}(q, p)$.

(iii). For any $\mathcal{M}$ over $\mathcal{L}$ and $X$ and for any $q, p \in Q$ and $x \in X^{*}$, $\delta_{x}^{*}(q, p)=\delta_{x}^{*}(p, q)$.

\section{Definition 3.3}

Let $\mathcal{M}_{1}=\left(Q_{1}, \delta_{1}, \sigma_{1}, \tau_{1}\right)$ and $\mathcal{M}_{2}=\left(Q_{2}, \delta_{2}, \sigma_{2}, \tau_{2}\right)$ be fuzzy finite automata over $\mathcal{L}$ and $X$. A (strong) homomorphism from $\mathcal{M}_{1}$ and $\mathcal{M}_{2}$ is a pair $(\alpha, \beta)$ of mappings $\alpha: \mathcal{M}_{1} \rightarrow \mathcal{M}_{2}$ and $\beta: X_{1} \rightarrow X_{2}$, 
such that $\delta_{1}(q, x, p)\left(=_{\mathcal{L}}\right) \leq_{\mathcal{L}} \delta_{2}(\alpha(q), \beta(x), \alpha(p))$ for any $q, p \in$ $Q_{1}$ and $x \in X_{1}$.

Theorem 3.4 Let $\mathcal{M}_{1}=\left(Q_{1}, \delta_{1}, \sigma_{1}, \tau_{1}\right)$ be a commutative fuzzy finite automaton over $\mathcal{L}$ and $X$, and $\mathcal{M}_{2}=\left(Q_{2}, \delta_{2}, \sigma_{2}, \tau_{2}\right)$ be $a$ fuzzy finite automaton over $\mathcal{L}$ and $X$. Let $(\alpha, \beta): \mathcal{M}_{1} \rightarrow \mathcal{M}_{2}$ be an onto strong homomorphism. Then $\mathcal{M}_{2}$ is commutative fuzzy finite automata over $\mathcal{L}$ and $X$.

\section{Definition 3.5}

Let $\mathcal{M}_{1}=\left(Q_{1}, \delta_{1}, \sigma_{1}, \tau_{1}\right)$ and $\mathcal{M}_{2}=\left(Q_{2}, \delta_{2}, \sigma_{2}, \tau_{2}\right)$ be fuzzy finite automata over $\mathcal{L}$ and $X$. A reverse homomorphism from $\mathcal{M}_{1}$ and $\mathcal{M}_{2}$ is a pair $(\alpha, \beta)$ of mappings $\alpha: \mathcal{M}_{1} \rightarrow \mathcal{M}_{2}$ and $\beta: X_{1} \rightarrow X_{2}$, such that

$$
\begin{aligned}
& \delta_{2}(\alpha(q), \beta(x), \alpha(p)) \\
& =\bigvee\left\{\delta_{1}(s, x, t) \mid s, t \in Q_{1}, \alpha(t)=\alpha(q), \alpha(s)=\alpha(p)\right\}
\end{aligned}
$$

for any $q, p \in Q_{1}$ and $x \in X_{1}$.

Theorem 3.6 Let $\mathcal{M}_{1}=\left(Q_{1}, \delta_{1}, \sigma_{1}, \tau_{1}\right)$ be a fuzzy finite switchboard automaton over $\mathcal{L}$ and $X$, and $\mathcal{M}_{2}=\left(Q_{2}, \delta_{2}, \sigma_{2}, \tau_{2}\right)$ be a fuzzy finite automaton over $\mathcal{L}$ and $X$. Let $(\alpha, \beta): \mathcal{M}_{1} \rightarrow \mathcal{M}_{2}$ be an onto reverse homomorphism. Then $\mathcal{M}_{2}$ is fuzzy finite switchboard automata over $\mathcal{L}$ and $X$.

\section{Fuzzy Finite Switchboard Subsystem}

\section{Definition 4.1}

Let over $\mathcal{M}=(Q, \delta, \sigma, \tau)$ be a fuzzy finite automaton over $\mathcal{L}$ and $X$. Let $\mu$ be a fuzzy subset of $Q$. Then $\mu$ is a fuzzy finite switchboard subsystem of $\mathcal{M}$, if

and

$$
\mu(q) \otimes \delta_{x}(q, p) \leq \mu(p)
$$

$$
\mu(q) \otimes \delta_{x y}(q, p) \leq \mu(p)
$$

for all $p, q \in Q$ and $x, y \in X$.

Theorem 4.1 The collection $\mathcal{S}(\mathcal{M})$ of all fuzzy finite switchboard subsystems of a fuzzy finite switchboard automata $\mathcal{M}$ forms a complete $\mathcal{L}$-sublattice of $\mathcal{F}_{\otimes}(Q)$.

Proof: It is very clear that the well-defined collection of $\mathcal{S}(\mathcal{M})$ of all fuzzy finite switchboard subsystems can be satisfied both reverse and commutative by definition 3.1 and 4.1 and theorem 3.2. Moreover, it is easy to check that the set of all fuzzy finite switchboard subsystems of a fuzzy finite switchboard automaton is closed under arbitrary meets, joins and $\emptyset$ and $Q$ are belongs to $\mathcal{S}(\mathcal{M})$. Thus $\mathcal{S}(\mathcal{M})$ is a complete $\mathcal{L}$-sublattice of $\mathcal{F}_{\otimes}(Q)$.

\section{Algorithm with CRL}

In order to enhance the performance, the algorithm of FFSA by using CRL is provided as below:

\subsection{Algorithm}

1. Enter the input in FFA and the output in FFSA.

2. Here, the $\sum$ consists of unit interval $[0,1]$.

3. Define the next states and every state for alphabet 0 and 1 .

4. $q_{0} \in Q$ the initial state is taken as input.

5. Check the validity of fuzzy switching automata and fuzzy commutative automata. If $\delta_{x}(q, p)=\left(\delta_{x}\right)(p, q)$ for $\forall q, p \in$ $Q$ and $x \in X$.then it is switching. If $\delta_{x y}(p, q)=\delta_{y x}(p, q)$ for $\forall q, p \in Q$ and $x, y \in X$, thus it is commutative.

Note: some of the states must satisfy the condition of switching state machine and commutative state machine. If the states not satisfy the both conditions, the states an usual state.
6. Find the membership value for each state by using CRL.

6.1 Suppose that $q \rightarrow^{x} p$ as a transition, where $p, q \in Q$ and $x \in X$. The values are given to each input symbols.

6.2 By using CRL, substitute the value of $x$ and $y$ regarding the path that are chosen to obtain the membership value.

6.3 Repeat the step 6.2 to obtain new membership value for another path.

7. Choose the best path or transition state based on the membership values.

\subsection{Algorithm construction of the fuzzy finite} switchboard automata by using CRL

Input: the set $Q$ of ( $n$ states) states of FFSA $\mathcal{M}=(Q, \delta, \sigma, \tau)$; the set $X$ of inputs alphabets of $\mathcal{M}$; the transition table $\delta$ of $\mathcal{M}=$

Output: YES, if $\mathcal{M}$ is FFSA with $n$ states, or NO, if $\mathcal{M}$ is not FFSA.

Procedure:

Step 1: Enter the state transition matrices $\delta_{x_{1}}, \delta_{x_{2}}, \ldots, \delta_{x_{n}}$.

Step 2: Set $i$ be the initial value, $i=1$ and $n \geq 2$.

Step 3: State transition $\delta_{x}, \delta_{y}, \ldots, \delta_{n}$. where $0 \leq \delta_{n} \leq 1$ denoted as the grade of membership of state transition. Step 4: If the transition has more than one membership value, enter the equation of CRL which is $\min \left(1-\delta_{x}+\right.$ $\left.\delta_{y}, 1\right)$ where $\left.0 \leq \delta_{x}, \delta_{y} \leq 1\right)$.

Step 5: Calculate the membership value by using $\min \left(1-\delta_{x}+\delta_{y}, 1\right)$.

- $\quad$ If $\delta_{x_{i}}(p, q) \geq 0.5$, then STOP, the output is the best path;

If $\delta_{x_{i}}(p, q) \leq 0.5$ then recalculate $\delta_{x_{i+1}}(p, q)$ and so on;

- If $\delta_{x_{i}}(p, q)=0$, the path is not chosen.

Step 6: for $i=i+1$ repeat Step 5 .

Step 7: for $i \leq n$, determine all possible path and calculate the membership value by using CRL

Step 8: for $i \leq n-1$, calculate $\delta_{x_{i}} \delta_{x_{i+1}}$ and $\delta_{x_{i+1}} \delta_{x_{i}}$

- If $\delta_{x_{i}} \delta_{x_{i+1}} \neq \delta_{x_{i+1}} \delta_{x_{i}}$ then STOP, the output $\mathcal{M}$ is not commutative; If $\delta_{x_{i}} \delta_{x_{i+2}}=\delta_{x_{i+2}} \delta_{x_{i}}$, recalculate $\delta_{x_{i}} \delta_{x_{i+2}}$ and $\delta_{x_{i+2}} \delta_{x_{i}}$;

If both are not equal then STOP, the output $\mathcal{M}$ is not commutative, NO;

Otherwise, recalculate $\delta_{x_{i}} \delta_{x_{i+3}}$ and

$\delta_{x_{i+3}} \delta_{x_{i}}$ and so on;

- If necessary, calculate until $\delta_{x_{i}} \delta_{x_{n}}$ and $\delta_{x_{n}} \delta_{x_{i}}$

If both are not equal, the output $\mathcal{M}$ is not commutative, $\mathrm{NO}$

If both are equal Go to Step 4 .

Step 9: $i=i+1$ repeat Step 8 .

Step 10: $i=n$, STOP, the output $\mathcal{M}$ is commutative, YES.

Step 11: for $i \leq n$, calculate $\delta_{x_{i}}(q, p)$ and $\delta_{x_{i}}(p, q) \forall p, q \in Q$.

- If $\delta_{x_{i}}(q, p) \neq \delta_{x_{i}}(p, q)$, then STOP, the output $\mathcal{M}$ is not switching;

If $\delta_{x_{i}}(q, p) \neq \delta_{x_{i}}(p, q)$, recalculate $\delta_{x_{i+1}}(q, p)$ and $\delta_{x_{i+1}}(p, q)$; 
- If both are not equal then STOP, the output $\mathcal{M}$ is not switching, NO;

- Otherwise, recalculate $\delta_{x_{i+2}}(q, p)$ and $\delta_{x_{i+2}}(p, q), ;$ and so on;

- If both are not equal, the output $\mathcal{M}$ is not switching, NO;

- $\quad$ If both are equal Go to Step 7.

Step 12: $i=i+1$ repeat Step 11 .

Step 13: $i=n$, STOP, the output $\mathcal{M}$ is switching, YES.

\subsubsection{Example}

Consider a FFSA system over $\mathcal{L}$ and $X$ as given below. Let $\mathcal{M}=$ $(Q, \delta, \sigma, \tau)$ where

a) $Q$ is a non-empty set, called the finite set of states,

b) $\delta: Q \times X \times Q \rightarrow L$ is a fuzzy subset of $Q \times X \times Q$, called the fuzzy transition function,

c) $\sigma: Q \rightarrow L$ is a fuzzy subsets of $Q$, called the fuzzy set of initial states,

d) $\tau: Q \rightarrow L$ is a fuzzy subsets of $Q$, called the fuzzy set of terminal states.

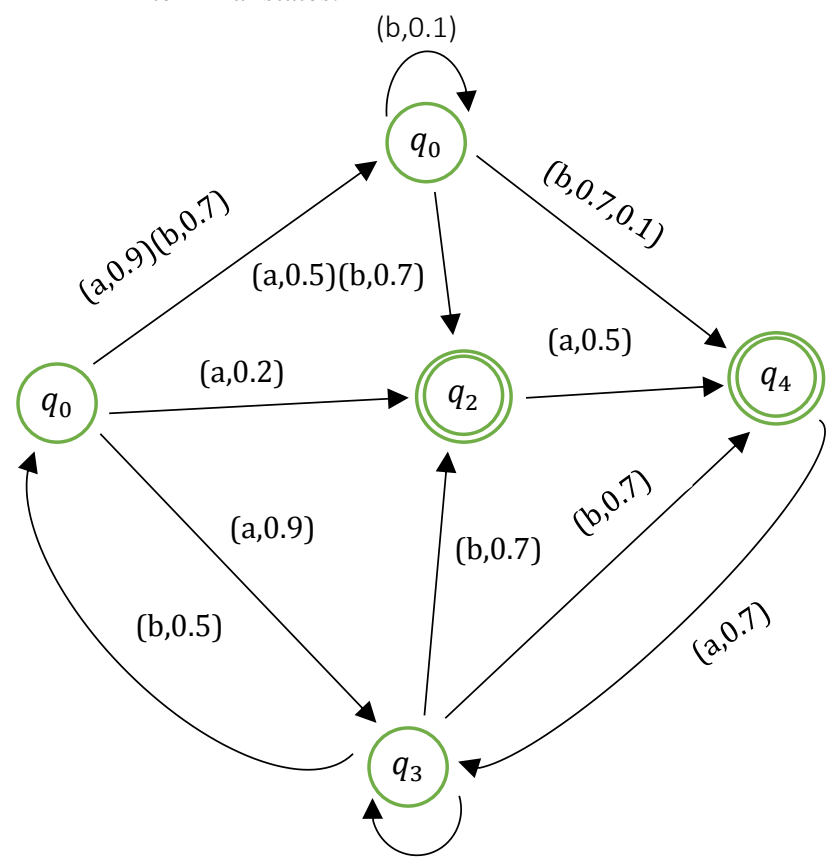

$(b, 0.1)$

Fig. 1: The system of Fuzzy Finite Switchboard Automata (FFSA)

Note that, $q \rightarrow^{x} p$ as a transition, where $q, p \in Q$ and $x \in X$. From Figure $1,=\left\{q_{0}, q_{1}, q_{2}, q_{3}, q_{4}\right\}, \delta=\{a, b\}$, and it consist the membership values in an arbitrary set with two distinguished elements which values in $\mathcal{L}$. According to the Figure 1, there are many paths we can pass through from one state to another state. For instance, from $q_{0}$ to $q_{2}$ there are 2 paths which are $q_{0} \rightarrow$ $q_{1} \rightarrow q_{2}$ or $q_{0} \rightarrow q_{3} \rightarrow q_{2}$ and for each, the input symbols are the same that is $a$ and $b$. Other example is from $q_{0}$ to $\mathrm{q}_{4}$ where there have many possibilities of path which are $q_{0} \rightarrow^{a} q_{1} \rightarrow^{b} q_{4}$ or $q_{0} \rightarrow^{a} q_{3} \rightarrow^{b} q_{4}$ but have different membership values. It also can be apply to the other states.

The system is switching if $\boldsymbol{\delta}_{\boldsymbol{x}}(\boldsymbol{q}, \boldsymbol{p})=\left(\boldsymbol{\delta}_{\boldsymbol{x}}\right)(\boldsymbol{p}, \boldsymbol{q})$ for $\forall \boldsymbol{q}, \boldsymbol{p} \in \boldsymbol{Q}$ and $\boldsymbol{x} \in \boldsymbol{X}$.

From Figure $1, \delta_{a b}\left(q_{0}, q_{4}\right)=\delta_{a b}\left(q_{4}, q_{0}\right)$, therefore the Figure 1 is switching.
If $\delta_{x y}(p, q)=\delta_{y x}(p, q)$ for $\forall q, p \in Q$ and $x, y \in X$. then $\mathcal{M}$ is called commutative.

Figure 1 is referred. Assume $\delta_{a b}\left(q_{0}, q_{2}\right)=\delta_{b a}\left(q_{0}, q_{2}\right)$, thus the Figure 1 is commutative. As a conclusion, Figure 1 is finite switchboard automata.

\subsubsection{Illustration of path calculation by using complete residuated lattice}

The equation of CRL is given by $x \rightarrow_{L} y=\min (1-x+y, 1)$

Table 1: The path calculation by using CRL

\begin{tabular}{|c|}
\hline Path \\
\hline$q_{0} \rightarrow^{a} q_{1} \rightarrow^{b} q_{2}$ or $q_{0} \rightarrow^{a} q_{3} \rightarrow^{b} q_{2}$ \\
\hline$q_{0} \rightarrow^{a} q_{2}$ \\
\hline$q_{0} \rightarrow^{a} q_{1} \rightarrow^{b} q_{4}$ or $q_{0} \rightarrow^{a} q_{3} \rightarrow^{b} q_{4}$ \\
\hline$q_{0} \rightarrow^{a} q_{1} \rightarrow^{b} q_{4}$ or $q_{0} \rightarrow^{a} q_{3} \rightarrow^{b} q_{4}$ \\
\hline$q_{0} \rightarrow^{a} q_{2} \rightarrow^{a} q_{4}$ \\
\hline$q_{0} \rightarrow^{a} q_{3}$ \\
\hline$q_{0} \rightarrow^{a} q_{1}$ \\
\hline$q_{0} \rightarrow^{a} q_{1} \rightarrow^{b} q_{1}$ \\
\hline$q_{0} \rightarrow^{a} q_{3} \rightarrow^{b} q_{3}$ \\
\hline
\end{tabular}

Table 1 shows the calculation of paths by using CRL. The values that are obtained by using CRL represent the probability of the path that might be chosen. For example, in Figure 1 there are many possibilities path from $q_{0}$ to $\mathrm{q}_{4}$. Therefore, the values that are nearest to 1 is assume as the best path. Regarding to the Figure 1 , from state $q_{0}$ to $\mathrm{q}_{4}$ when the input symbol is $a$, $\mathrm{q}_{0} \rightarrow{ }^{\mathrm{a}} \mathrm{q}_{2} \rightarrow{ }^{\mathrm{a}} \mathrm{q}_{4}$ the truth value is 1 that show it is the best choice of path. This figure below is the selected path according to the membership values.

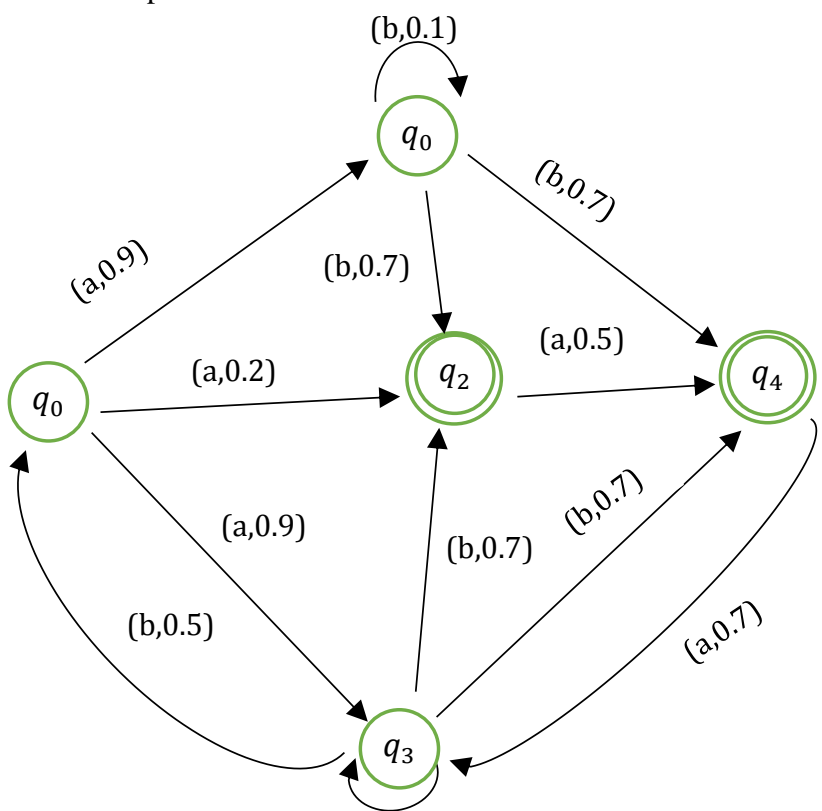

(b,0.1)

Fig. 2: The simplest system after consider membership value by CRL

\section{Conclusion}

In a conclusion, a notion of fuzzy finite switchboard automata (FFSA) with complete residuated lattices is proposed. If the system fulfilled the two properties which are fuzzy commutative automata and fuzzy switching automata, it can be called as finite switchboard automata. Complete Residuated Lattices (CRL) are 
important general algebraic that have close links with various important logics [15], branches of logic and linear logic [16]. To make it more easy, the algorithm of CRL is developed and an example is provided. By understanding the concept of FFSA, it might lead to a new invention and products. In future research, it would be interesting to study about fuzzy transformation semigroup based on switchboard concept by using CRL or other method of truth value.

\section{Acknowledgement}

This work was supported in part by Fundamental Research Grant Schemes (FRGS) under vot no1562, Ministry of Higher Education, Malaysia.

\section{References}

[1] Pan H, Cao Y, Zhang M \& Chen Y (2014), Simulation for latticevalued doubly labeled transitions systems. International Journal of Approximate Reasoning 55, 797-811.

[2] Konecny J \& Krupka M (2017), Complete relations on fuzzy complete lattices. Fuzzy Sets and Systems 320, 64-80.

[3] Sato Y \& Kuroki N (2002), Fuzzy Finite Switchboard State Machine. The Journal of Fuzzy Mathematics 10, 863-873.

[4] Ciric M, Ignjatovic J \& Damljanovi'c N (2012), Bisimulations for fuzzy automata. Fuzzy Sets and Systems 186, 100-139.

[5] Xing H, Qiu DW \& Liu F (2009), Automata theory based on complete residuated lattice-valued logic: pushdown. Fuzzy Sets and Systems 160, 1125-1140.

[6] Sato Y, Finite Switchboard State Machines and Fuzzy Finite Switchboard State Machines, Hyogo University of Teacher Education, (2003)

[7] Rabin M \& Scott D (1959), Finite Automata and Their Decision Problems. Ibm Journal of Research and Development 3, 114-125.

[8] Mordeson JN \& Malik DS, Fuzzy Automata and Languages, Theory and Applications, Chapman and Hall/CRC, London/Boca Raton, FL (2002).

[9] Wu L, Qiu DW \& Xing H (2012), Automata theory based on complete residuated lattice-valued logic: turing machines. Fuzzy Sets and Systems 208, 43-66.

[10] Ignjatovic J, Ciric M \& Simovic V (2013), Fuzzy relation equations and subsytems of fuzzy transition systems. Knowledge-Based Systems 38, 48-61.

[11] Guo X (2012), A comment on "Automata theory based on complete residuated lattice-valued logic: Pushdown automata". Fuzzy Sets and Systems 199, 130-135.

[12] Tabak J, Algebra Sets, Symbols, and the Language of Thought, The History of Mathematics, Volume 1, Viva Books Private Limited, (2006).

[13] Pan H, Li Y, Cao Y \& Li P (2017), Nondeterministic fuzzy automata with membership values in complete residuated lattices, International Journal of Approximate Reasoning 82, 22-38.

[14] Abolpour K \& Zahedi MM (2017), General fuzzy automata based on complete residuated lattice-valued. Journal of Fuzzy Systems 14, 103-121.

[15] Qiu DW (2001), Automata theory based on completed residuated lattice-valued logic(I), Science in China Series: Information Sciences 44, 419-429.

[16] Dilworth RP (1938), Abstract residuation over lattices. Bulletin of the American Mathematical Society 44, 262-268.

[17] Wu L \& Qiu DW (2010), Automata theory based on complete residuated lattice-valued logic: reduction and minimization. Fuzzy Sets and Systems 161, 1635-1656.

[18] Belohlavek R, Fuzzy Relational Systems: Foundations and Principles, Kluwer, (2002)

[19] Ghorani M \& Zahedi MM (2012), Characterizations of complete residuated lattice-valued finite tree automata. Fuzzy Sets and Systems 199, 28-46.

[20] Qiu DW (2002), Automata theory based on completed residuated lattice- valued logic(II). Science in China Series:Information Sciences 45, 442-452.

[21] Ciric M, Ignjatovic J \& Pethovic T (2010), Fuzzy relation equation and reduction of fuzzy automata. Journal Computer System Science 76, 609-633.
[22] Li Y (2011), Finite Automata Theory with Membership values in lattices. Information Sciences 181, 1003 - 1017.

[23] Wee WG, On generalization of adaptive algorithm and application of the fuzzy sets concept

[24] to pattern classification, Ph.D. Thesis, Purdue University, Lafayette, IN, (1967).

[25] Li YM \& Pedrycz W (2005), Fuzzy finite automata and fuzzy regular expressions with membership values in lattice-ordered monoids. Fuzzy Sets and Systems 156, 68-92. 